\title{
Infant Warmer Design with PID Control for Stability and Equal Temperature Distribution Equipped with Digital Scales for Prevention of Hypothermia in Newborns
}

\author{
Ishika Sharma and Monika Singh \\ NITTTR, Chandigarh, India \\ Corresponding author: Monika Singh (e-mail: Monika2002@gmail.com).
}

ABSTRACT Babies need temperatures that match the temperature in the mother's womb, which is between $35^{\circ} \mathrm{C}-37^{\circ} \mathrm{C}$. the latest research on infant warmer equipment uses the fuzzy method as a system for temperature control in infant warmers, The problem in previous research is that at each temperature setting, the temperature is not evenly distributed throughout the bed, when it reaches the setting temperature, the heater continues to turn on so that the bed gets hotter. The purpose of this research is to make an infant warmer equipped with digital scales, with temperature settings of $35^{\circ} \mathrm{C}, 36^{\circ} \mathrm{C}$, and $37^{\circ} \mathrm{C}$ using PID control to stabilize the temperature and ensure the spread of heat on the bed evenly, then the addition of skin temperature aims to make nurses know what the patient's body temperature is when observations were made. The infant warmer in this module uses an Arduino microcontroller which is displayed in 7segment, then the skin sensor used is the DS18B20 temperature sensor to read the skin temperature, and the infant warmer temperature sensor used is LM35 as a PID control system. The results of the research in making the tool module were compared with the measurement results against the comparator. This research has obtained the results of the smallest error in the measurement of setting temperature of $35^{\circ} \mathrm{C}$ with an error of $0 \%$. For comparison with the incu analyzer, the smallest error is obtained at the setting temperature of $37^{\circ} \mathrm{C}$ with an error value of $0 \%$ on the T5 measurement, the difference in skin temperature against the thermometer is $0.1^{\circ} \mathrm{C}$. The results showed that the temperature spread on the module had different error values. So that this research can be implemented on the PID control infant warmer system to improve performance on infant temperature stability.

INDEX TERMS Infant Warmer, LM35, DS18B20, PID Control, 7 Segment

\section{INTRODUCTION}

After the baby is born, the temperature of the baby's internal body and skin can decrease by about $0.1^{\circ} \mathrm{C}-0.3^{\circ} \mathrm{C}$ per minute, from this incident it can still be prevented by therapeutic measures using an infant warmer as the first treatment after the baby is born[1]. Hypothermia or lack of body temperature below normal limits is a significant problem that often occurs in newborns[2]. There are more than 20 million premature and low birth weight babies born every year, and $95 \%$ of premature and low birth weight babies are born in developing countries. There are 3 million babies who die in the first 28 days after birth[2].

In the neonatal period, infants have the highest risk of health problems, because the baby's body is still vulnerable 
[3].Infant warmer is a life support equipment that is used to provide relief from the effects of heat on normal babies and premature babies who are unable to maintain their own body temperature when in a new environment. In the care of normal and premature babies, GE health care makes infant warmer equipment, including panda warmer and giraffe warmer who were placed in neonatal intensive care units (NICU).[4]

Furthermore, the research was redeveloped by Brahminindya Resi Kanastriloka and Maimunah Novita Sari in 2018 with the title Infant Warmer equipped with phototherapy. The tool uses Arduino Uno as a data processor and then uses an LCD as a temperature display. The weakness of this tool is that the LCD is used because the user is difficult to see the temperature indicator on the LCD, must come closer, the mechanics of the phototherapy lamp are too rough so it is difficult to move, using a digital sensor to reduce the temperature reading error value [5]. In the research conducted by Anggraeni Dara Pratiwi with the title infant incubator based on proportional integral and derivative equipped with kangaroo mode researchers use a PID control system which is also combined with kangaroo mode, using a minimum system ATMega 328 which processes analog data from the LM 35 and the Thermistor sensor with a temperature setting of $32^{\circ} \mathrm{C}$ to $37^{\circ} \mathrm{C}$. In this tool, there are still weaknesses in the PID response to heater performance because the heater wattage is too small so the PID response is a little slow [6]. Subsequent research was carried out by I. W. Aris Wiyadnyana Putra, W. Widhiada, and I. N. Suarnadwipa with the title PID system for control of temperature and humidity stability in an arduino microcontroller-based infant incubator. This tool uses a PID system with a trial and error tunning method using a DHT 22 sensor and an LM 35 sensor as a skin sensor. The drawback in this study is that the DHT 22 sensor is less sensitive than other sensors [7]. Then in Iraq, a smart incubator based on PID controller The device used a DHT11 sensor as a temperature sensor and an LM 35 sensor as a skin sensor, with PID control [8]. which uses the C programming language with IDE software and is processed on Arduino Mega. Economical Control System In Power Consumption. The research entitled PID temperature controller infant incubator using RTD explains that the PID algorithm method that controls PWM to control the heater [9] to keep the heater at the desired temperature setting, uses the PT100 RTD sensor. However, this tool has additional parameters that are still lacking to observe in infants [10]. In 2012 further research was carried out by Sulistya Anggara Wira bhuwana with the title digital baby warmer equipped with phototherapy unit The tool is still using a fuzzy logic system, The drawback in this study is that the fuzzy logic method is still not precise for its control and is still not equipped with scales for observation of the baby[11]. This study aims to create and design an infant warmer with a DC heater equipped with a battery so that in the event of a power outage by PLN this tool can still be used. the weakness of this tool is too much battery[12]. This research use DHT11 sensor as sensing element and the control method are actuated by lightbulbs. The controller used microcontroller atmega16 as processor which output determines the amount of power supplied to the lightbulb by employing pulse width modulation and MOSFET triggered circuit[13]. The apgar score provides an accepted and convenient method for reporting the status of the newborn infant immediately after birth and the response to resuscitation if needed[14]. the author modified one of the Infant Warmer tools in the NICU RSAL Dr. Ramelan is used to provide the first care for newborns in the form of heating and therapy for infants with hyperbilirubinemia. the weakness of the skin sensor reading is still not accurate[15]. The objective of the work is to interface Programmable ADT7410 DTS to PIC18F8720 microcontroller to monitor the infants body temperature[16]. This sensor measures temperature with accuracy, high resolution and fast conversion[17]. Proposed system design considers the precision control for Sensitive Application[18] Which is achieved using PWM calculated with PID Algorithm[19]. To control temperature stability at $36^{\circ} \mathrm{C}$ and humidity at $80 \%-60 \% \mathrm{RH}$ value in incubator space required arduino, sensor, and added PID control (Proportional Integral Derivative) to reduce maximum overshoot (Mp) and error signal average $\leq 5 \%$ and speed up the system to reach the setting point. With the PID control the maximum overshoot value $\mathrm{Mp}=0.833889 \%[20]$.

Based on the results of the identification of problems that exist from existing research, the authors want to develop and overcome these shortcomings by making a final project research using the LM35 sensor for infant temperature and the DS18B20 sensor for skin temperature, entitled Infant warmer with digital scales (infant warmer PID control). The purpose of this research is to make an infant warmer equipped with digital scales. With a temperature setting of 35 to $37^{\circ} \mathrm{C}$ using PID control to stabilize the temperature and ensure an even distribution of heat on the bed. as well as the addition of an APGAR Timer up to 20 minutes and the addition of a digital scale for easy observation of babies. Then the addition of skin temperature aims to let nurses know what the patient's body temperature is when observing.

\section{MATERIALS AND METHODS \\ A. EXPERIMENTAL SETUP}

This study uses measurements of temperature values in the range of $35^{\circ} \mathrm{C}, 36^{\circ} \mathrm{C}$ and $37^{\circ} \mathrm{C}$ by taking measurements at T1, T2, T3.T4, and T5 taken 10 times for data collection.

\section{1) MATERIAL AND TOOL}

This research uses AT Mega 2560 and Arduino Uno microcontroller as data processing. The output of the microcontroller is a display on the 7 segment cathode as a display. This study uses the LM35 sensor as the room temperature reading and the DS18B20 sensor as the patient's skin temperature reading. by using a 1200 -watt power heater element 


\section{2) EXPERIMENT}

In this research, after the design is complete, the LM35 sensor will be compared with the incu analyzer with 10 data retrieval within 1 hour, and the DS18B20 sensor compared to a contact body temperature thermometer with 6 times data collection on the human body.

\section{B. THE DIAGRAM BLOCK}

The microcontroller is used to process the temperature and time settings that have been inputted as temperature controller settings. The data will be sent to the microcontroller and then forced to control the heater according to the temperature that has been set between $35^{\circ} \mathrm{C}$ - $37^{\circ} \mathrm{C}$. For infant warmers, First select the desired temperature setting by pressing the setting button. Then the start button indicates that the Microcontroller has started processing the work of the infant warmer. Reset button is used to stop program performance and sensor readings, The APGAR timer was used to assign the newborn's APGAR score. If the Room Temperature Sensor does not read the temperature or the temperature is excessive, the thermostat will turn off the heater when it reaches the thermostat setting temperature. These steps can be seen in accordance in FIGURE 1 .

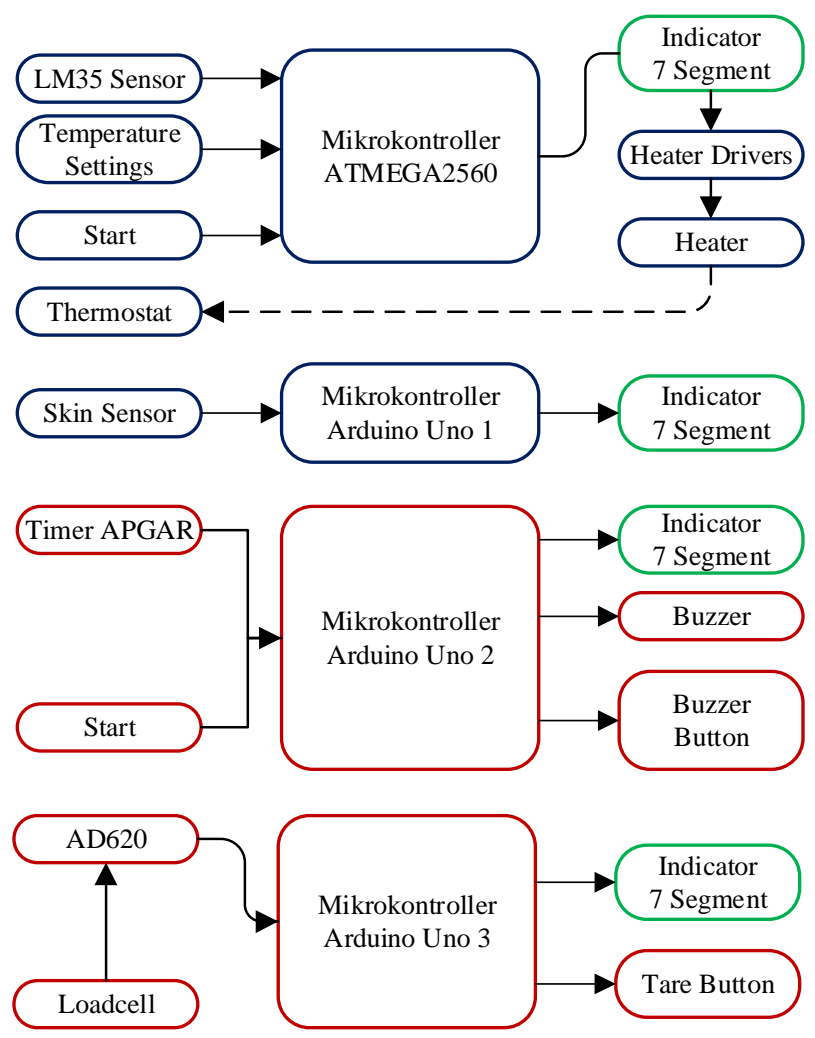

FIGURE 1. Diagram Block

\section{THE FLOWCHART}

The control program was built based on the flowchart as shown in FIGURE 2. At start (start) the microcontroller performs initialization and then the user sets the temperature so that the microcontroller detects the value of the difference between the set point temperature and the actual temperature, the difference in value will be input to the PID control. The PID control processes the error value and determines the output value for the heater. for the PID process itself works simultaneously for the Proportional function to quickly get the V set, for Integral to speed up the work response and derivatives to reduce the response or hold and reduce the ripple to be stable. let's say the actual temperature is $25^{\circ} \mathrm{C}$ and the desired temperature is $35^{\circ} \mathrm{C}$. when the actual temperature has not reached the setting temperature it will be feedback to the PID so that the PID output will control the heater driver until the setting temperature is reached and stabilizes it. Then the temperature sensor will read the actual temperature value and compare it with the setting temperature. Then the temperature sensor will read the actual temperature value and compare it with the setting temperature. The PID control will continue to process the error value and determine the output value so that the actual temperature is the same as the setting temperature (error value $=0$ ). If the temperature is stable, the APGAR timer is activated by the user, the APGAR timer buzzer sounds at $1,5,10,15$, and 20 minutes the buzzer is turned off using a reset alarm, if it is finished then the process ends.

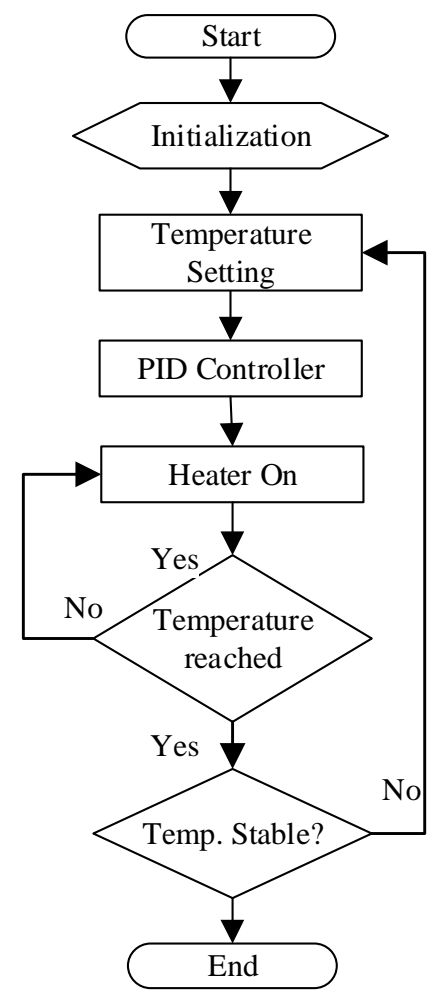

FIGURE 2. The Flowchart of PID Control 


\section{THE ANALOG CIRCUIT}

The base leg of the transistor is controlled using a microcontroller when given logic 1 then it turns on the 7segment command, then the 7segment leg of a,b,c,d,e,f,g is controlled directly by the microcontroller, when segment a is given logic 0 then it turns on segment a. The 7 segment circuit is a circuit to drive 7 segment so that it can be controlled by the microcontroller easily.

The intended solenoid valve driver is used for the selection of flow rate, occlusion and drain modes. Each solenoid valve will be connected to a series of solenoid valves. The initial condition of solenoid valve is Normally Close so that when given solenoid voltage the valve will open.

The output issued by the DS1820 sensor is a configuration of numbers 1 and 0 , which indicates a certain temperature. The amount of data bit output voltage also depends on the amount of voltage that is input to the sensor. This ds $18 \mathrm{~b} 20$ sensor circuit requires a pullup resistor to be able to read the data value from the DS18B20 sensor in order to display temperature data.

\section{RESULT}

In this study, the function test was carried out directly with a comparison tool using the Incu Analyzer II (Fluke), with testing the DS18B20 sensor using a contact thermometer comparison (Omron). The results of the data collection show that the results are suitable for use in patients who need an infant warmer. The proposed design is shown in FIGURE 3 and FIGURE 4. FIGURE 4 is infant warmer box design.

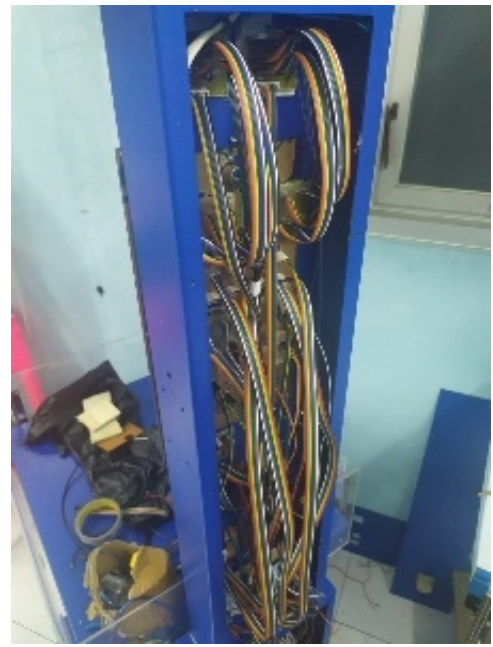

FIGURE 3. The Analog Digital Part

\section{1) DESIGN MODULE}

Photo of the analog and digital parts of the infant warmer shown in FIGURE 4 for analog consists of an LM35 sensor, a DS18B20 sensor and a 7 segment circuit. in analog circuit there is a pull up resistor for the DS18B20 sensor and a seven-segment circuit using a transistor then for the digital components Arduino uno and Arduino mega as processors of a controller. so that the PID process occurs in a program. and in FIGURE 6 is the front view of the infant warmer box design.

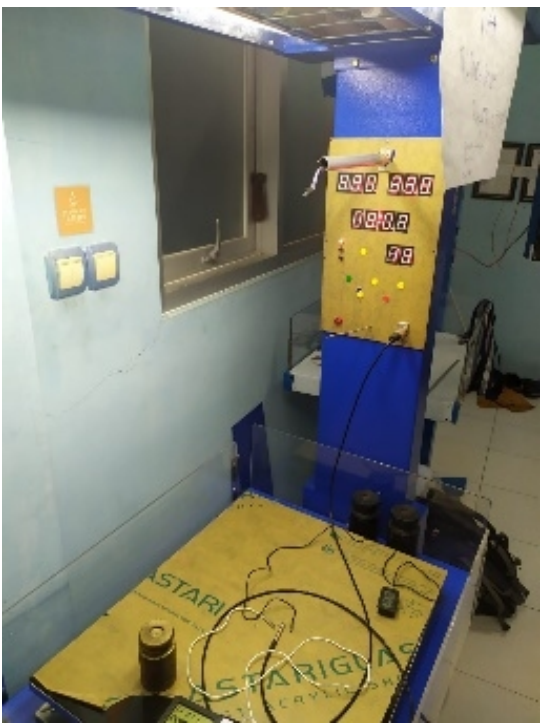

FIGURE 4. Design Modul

\section{2) THE LISTING PROGRAM FOR ARDUINO}

Pseudocode: 1. Program Temperature Setting.

\begin{tabular}{|c|c|}
\hline 1. & void setsuhu()\{ \\
\hline 2. & IF $($ digitalRead $(7)==\mathrm{HIGH})\{$ \\
\hline 3. & $\mathrm{a}++$ \\
\hline 4. & delay(100); \\
\hline 5. & \} \\
\hline 6. & $\operatorname{IF}(a==3)\{$ \\
\hline 7. & $\mathrm{a}=0$ \\
\hline 8. & \} \\
\hline 9. & IF $(a==0)\{$ \\
\hline 10. & tigalima(); \\
\hline 11. & \} \\
\hline 12 & IF $(a==1)\{$ \\
\hline 13. & tigaenam(); \\
\hline 14. & \} \\
\hline 15. & IF $(a==2)\{$ \\
\hline 16. & tigatujuh(); \\
\hline 17. & \} \\
\hline 18. & void tigalima()\{ \\
\hline 19. & digitalWrite $(10,1)$ \\
\hline 20. & digitalWrite $(11,0)$; \\
\hline 21. & digitalWrite $(12,0)$ \\
\hline 22. & setpoint $=50.0$ \\
\hline 23. & \} \\
\hline 24. & void tigaenam ()\{ \\
\hline & digitalWrite $(11,1)$; \\
\hline 25. & digitalWrite $(10,0)$; \\
\hline
\end{tabular}




\begin{tabular}{l|l} 
26. & digitalWrite $(12,0) ;$ \\
27. & setpoint $=51.0 ;$ \\
28. & void tigatujuh ()\{ \\
29. & digitalWrite $(12,1) ;$ \\
30. & digitalWrite $(11,0) ;$ \\
31. & digitalWrite $(10,0) ;$ \\
32. & setpoint $=52.0 ;$ \\
33. & \}
\end{tabular}

After the device is ON, the initial display on the display is the skin temperature, room temperature and the temperature setting indicator, then press the temperature setting to start the selection at what temperature point you want in the required observation process. In this tool the temperature setting ranges from $35^{\circ} \mathrm{C}$ to $37^{\circ} \mathrm{C}$. show in Pseudocode: 1 .

Pseudocode: 2. Program Sensor LM35.

\begin{tabular}{|l|l|}
\hline 1. & \#define LM35_pin A0 \\
2. & int temp; \\
3. & temp $=10 *$ analogRead(LM35_pin $) / 9.3 ; / /$ read \\
4. & analog voltage and convert it to ${ }^{\circ} \mathrm{C}(9.3=$ \\
5. & $1023 /(1.1 * 100))$ \\
6. & delay(1000); // wait 1 second
\end{tabular}

The above program is used to read the LM35 sensor, LM35 is an analog temperature sensor whose output is a voltage and then converted to Celsius for every $10 \mathrm{C}$ increase is $10 \mathrm{mV}$ for the LM35 pin, initialized to pin A0. show in Pseudocode: 2

Pseudocode: 3. Program Sensor DS18B20.

\begin{tabular}{l|l} 
1. & \#include <OneWire.h> \\
2. & \#include <DallasTemperature.h> \\
3. & \#define ONE_WIRE_BUS 2 \\
4. & suhu = sensors.getTempCByIndex $(0) ;$ \\
5. & temp = suhu; \\
6. & sensors.requestTemperatures ()$;$
\end{tabular}

Initialization in the temperature program contains the DS18B20 temperature sensor library which is a serial communication using one data line. Where the data/output leg of the DS18B20 temperature sensor is initialized at pin 2. show in Pseudocode: 3

Pseudocode: 4. PID program.

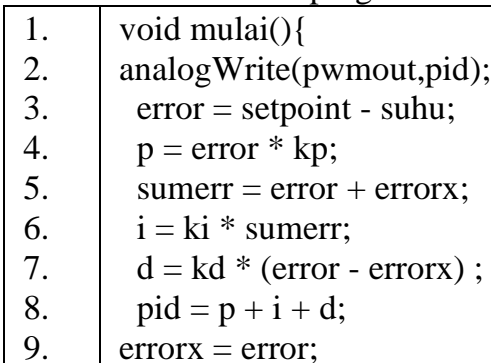

\begin{tabular}{l|l}
\hline 10. & $\mathrm{IF}($ pid $<1)\{$ \\
11. & pid $=0$ \\
12. & \} \\
13. & $\mathrm{IF}($ pid $>255)\{$ \\
14. & pid = 255; \\
15. & \} \\
16. & \}
\end{tabular}

The heater control using PID and PID will work when the range is 0 to 255. If the PID is less than 1 then the PID will still show 0 and if the PID is more than 255 then the PID will still show 255. This aims to limit if the PID shows a number ( - ) or above 255, before the PID control process occurs, there is a PID formula in the program, the error value is obtained from the setpoint minus the actual temperature, then $\mathrm{P}=$ the error value multiplied by the predetermined $\mathrm{Kp}$. The summer value is obtained from the error value added to the error value $\mathrm{x}$, so the error value $\mathrm{x}$ is the same as the value of the error value. Then $\mathrm{i}=\mathrm{Ki}$ multiplied by the value of summer. Then $\mathrm{d}=\mathrm{Kd}^{*}$ (error - errorx) after that the results of each PID are added up. show in Pseudocode: 4

\section{3) THE MEASUREMENT RESULT WITH INCU} ANALYZER

TABLE 1.

Temperature Setting $35^{\circ} \mathrm{C}$

\begin{tabular}{|c|c|c|c|c|c|c|}
\hline Mean & 34,28 & 34,21 & 34,84 & 33,73 & 34,99 & 35,0 \\
\hline SD & 0,13 & 0,11 & 0,26 & 0,04 & 0,08 & 0,07 \\
\hline \% Error & 0,02 & 0,02 & 0,01 & 0,03 & 0,02 & \\
\cline { 1 - 4 } UA & 0,04 & 0,03 & 0,08 & 0,01 & 0,02 & \\
\hline
\end{tabular}

From 10x temperature readings, the tool module reads the lowest temperature value at $34.9^{\circ} \mathrm{C}$ and the highest value at $35.1^{\circ} \mathrm{C}$. While the Incu Analyzer reads the lowest value at $\mathrm{T} 5$ at $34.81{ }^{\circ} \mathrm{C}$ and the highest value at $35.07^{\circ} \mathrm{C}$. The measurement result was showed in TABLE 2.

TABLE 2.

Temperature Setting $36^{\circ} \mathrm{C}$

\begin{tabular}{|c|l|l|l|l|l|l|}
\hline Mean & 34,56 & 34,84 & 35,60 & 34,30 & 36,10 & 36,06 \\
\hline SD & 0,18 & 0,04 & 0,13 & 0,21 & 0,05 & 0,09 \\
\hline \% Error & 0,04 & 0,03 & 0,01 & 0,04 & 0,01 & \\
\cline { 1 - 4 } UA & 0,05 & 0,01 & 0,04 & 0,06 & 0,01 & \\
\hline
\end{tabular}

The measurement result was showed in TABLE 2. From 10x temperature readings, the tool module reads the lowest temperature value at $35.9^{\circ} \mathrm{C}$ and the highest value at $36.2^{\circ} \mathrm{C}$. While the Incu Analyzer reads the lowest value at T5 of $35.99^{\circ} \mathrm{C}$ and the highest value at $36.17^{\circ} \mathrm{C}$ 
TABLE 3.

Temperature Setting $37^{\circ} \mathrm{C}$

\begin{tabular}{|c|l|l|l|l|l|l|}
\hline Mean & 35,52 & 35,07 & 35,88 & 34,81 & 37,07 & 37,01 \\
\hline SD & 0,24 & 0,04 & 0,29 & 0,24 & 0,07 & 0,07 \\
\hline \% Error & 0,04 & 0,05 & 0,03 & 0,06 & 0,00 & \\
\cline { 1 - 5 } UA & 0,07 & 0,01 & 0,09 & 0,07 & 0,02 & \\
\hline
\end{tabular}

From 10x temperature readings, the tool module reads the lowest temperature value at $36.9^{\circ} \mathrm{C}$ and the highest value at $37.1^{\circ} \mathrm{C}$. While the Incu Analyzer reads the lowest value at $\mathrm{T} 5$ at $36.96^{\circ} \mathrm{C}$ and the highest value at $37.18^{\circ} \mathrm{C}$. The measurement result was showed in TABLE 3.

\section{DISCUSSION}

Measurements on the module using an incu analyzer and a contact thermometer. Measurement tools are carried out 10 times in order to get constant results. The results of the measurement of the temperature setting, when we tested each temperature setting it had a different value. What happens in the $35^{\circ} \mathrm{C}$ setting test results in the error value in the tool module $0 \%$, T5 $0.02 \%$, T4 $0.03 \%$, T3 $0.01 \%$, T2 $0.02 \%, \mathrm{~T} 10.02 \%$ indicates that when setting the temperature $35^{\circ} \mathrm{C}$ voltage obtained $0.38 \mathrm{Vdc}$, the average on the module is around 35.00 . Then in the $36^{\circ} \mathrm{C}$ setting test, the error value in the tool module is $0.01 \%$, T5 $0.01 \%$, T4 $0.04 \%$, T3 $0.01 \%, \mathrm{~T} 20.03 \%$, and $\mathrm{T} 10.04 \%$ indicates that at the time of temperature setting $36^{\circ} \mathrm{C}$ voltage obtained 0.39 $\mathrm{Vdc}$, the average on the module is about 36.06. And in the test setting at $37^{\circ} \mathrm{C}$, the error value on the tool module is $0.01 \%$, T5 $0 \%$, T4 $0.06 \%$, T3 $0.03 \%$, T2 $0.05 \%$ and $\mathrm{T} 1$ $0.04 \%$ indicates that when setting the temperature to $37^{\circ} \mathrm{C}$ the voltage obtained is $0.40 \mathrm{Vdc}$, the average on the module is about 37.01. So when testing is done in an open room. And testing the skin temperature on the first measurement, the error between the module and the thermometer is 0.1 with an error value of $0.03 \%$. For the second measurement the error between the module and the thermometer is 0 with an error value of $0 \%$. The third measurement of the error between the module and the thermometer is 0.1 with an error value of $0.03 \%$. The fourth measurement of the error between the module and the thermometer is 0.1 with an error value of $0.03 \%$. The fifth measurement of the error between the module and the thermometer is 0.1 with an error value of $0.03 \%$. The sixth measurement of the error between the module and the thermometer is 0.2 with an error value of $0.05 \%$. So, the average error is about 0.1 with an average error value of $0.03 \%$.

For the author's research with previous studies, there are differences in results at each temperature setting and at skin temperature. For previous research [5] using the fuzzy method, which has the largest error value, at $\mathrm{T} 10.06 \%$ at a temperature setting of $34^{\circ} \mathrm{C}$, then $\mathrm{T} 10.06 \%$ at a temperature setting of $35^{\circ} \mathrm{C}$, and $\mathrm{T} 10.06 \%$ at a temperature setting of $36^{\circ} \mathrm{C}$, and $\mathrm{T} 10.07 \%$ at a temperature setting of $37^{\circ} \mathrm{C}$. While in this study using the PID control method, which has the largest error value, namely $\mathrm{T} 40.03 \%$ at a temperature setting of $35^{\circ} \mathrm{C}$, then $\mathrm{T} 40.04 \%$ at a temperature setting of $36^{\circ} \mathrm{C}$, and $\mathrm{T} 40.06 \%$ at a temperature setting of $37^{\circ} \mathrm{C}$. For the skin temperature of previous researchers [5], the average value of the difference between the module and the thermometer is around $0.6^{\circ} \mathrm{C}$. While this research has an average value of the difference between the module and the thermometer, which is $0.1^{\circ} \mathrm{C}$. From the results of the data that has been studied by previous researchers and the results of research from the author, it can be concluded that the author's research has a smaller error value and error value than previous researchers [5], so the benefits can be used this tool with an open room temperature with 3 temperature settings PID control which is equipped with a temperature sensor for the body using 7 segments that are clearly visible even from 2 meters of vision.

So it can be concluded that the tool module that has been compared with the calibrator has a weakness in the module on this tool, namely the distance to the sensor that is too close will affect the results of the heater control, and this time the tool still uses many microcontrollers and has not been equipped with phototherapy for therapy in infants.

\section{CONCLUSION}

Conclusion The purpose of this research is to make an infant warmer equipped with digital scales. With a temperature setting of 35 to $37^{\circ} \mathrm{C}$ using PID control to stabilize the temperature and ensure the spread of heat on the bed. This research has obtained the results of the smallest error in the measurement of the temperature setting of $35^{\circ} \mathrm{C}$ with an error of $0 \%$ and the result of the largest error at setting the temperature of 36 and $37^{\circ} \mathrm{C}$ with an error value of $0.01 \%$. For comparison with the incu analyzer, the smallest error was obtained at the setting temperature of $370 \mathrm{C}$ with an error value of $0 \%$ on the $\mathrm{T} 5$ measurement and the largest error at the setting temperature of $37^{\circ} \mathrm{C}$ with an error value of $0.06 \%$ on the T4 measurement. From the measurements that have been made, it can be concluded that the placement of the sensor distance to the heater can affect the control results on the heater driver, using a good driver can improve the heater control and temperature readings on the display and use a temperature sensor that can read the temperature linearly because it will affect the results.

\section{REFERENCES}

[1] E. M. Mccall, F. Alderdice, H. L. Halliday, S. Vohra, and L. Johnston, "Interventions to prevent hypothermia at birth in preterm and/or low birth weight infants," Cochrane Database Syst. Rev., vol. 2018, no. 2, 2018, doi: 10.1002/14651858.CD004210.pub5.

[2] World Health Organization, "Who compendium of innovative health technologies for low-resource settings 2011-2014.," Healtcare Jurnal., vol 2016, no 1, p. 143, 2016.

[3] I. K. O. Azizah, "Neonatal Death in Grobogan Kabupaten District," Higeia J. Public Heal. Res. Dev., vol. 1, no. 3, pp. 84-94, 2017, [Online]. Available: http://journal.unnes.ac.id/sju/index.php/higeia.

[4] H. S. Hutagaol, E. Darwin, and E. Yantri, "Effect of Early Initiation of Breastfeeding (IMD) on Temperature and Heat Loss in Newborns," J. Kesehat. Andalas, vol. 3, no. 3, pp. 332-338, 2014, 
doi: 10.25077/jka.v3i3.113.

[5] B. R. Kanastriloka, E. Yulianto, and A. Kholiq, "Infant Warmer Equipped With Phototherapy,” J.teknokes, vol2 no. 1, pp. 6-8, 2003, [Online]..

[6] A. D. Pratiwi, E. Yulianto, and A. Kholiq, "Proportional Integral and Derivative (PID) Based Infant Incubator Equipped With Kangaroo Mode," J. Teknokes, vol. 12, no. 1, pp. 33-38, 2019, doi: 10.35882/teknokes.v12i1.6.

[7] I. W. A. W. Putra, W. Widhiada, and I. N. Suarnadwipa, "PID System Control Temperature and Humidity Stability In Baby Incubator Based On Arduino Microcontroller," vol. 7, no. 3, pp. 245-249, 2018

[8] N. Z.A, Y. P. Roja, and N. Sylvia, "PID Control Application in Formic Acid Plant Reactor with Capacity of 100,000 Tons/Year," $J$. Teknol. Kim. Unimal, vol. 7, no. 2, p. 135, 2019, doi: 10.29103/jtku.v7i2.1253.

[9] A. Irsyad, Isnawaty, and R. A. Saputra, "Implementation of a Navigation System Using the Proportional Integral Derivative (Pid) Method on the Wall Follower Robot," semanTIK, vol. 3, no. 2, pp. 9-12, 2017.

[10] Z. S. A. Rahman and F. S. A. Hussain, "Smart Incubator Based on PID Controller," Int. Res. J. Eng. Technol., vol. 4, no. 3, pp. 25012509, 2017, doi: 10.13140/RG.2.2.21917.77282.

[11] Bhuwana, S.A.W. " Digital Infant Warmer Equipped with Phototherapy Unit (Apgar Timer and Temperature Control)," J. Eng. Technol, vol 6, no2. pp. 200-218, 2012

[12] B. Wahyudi, M. Miftahudin, and I. Firdaus, "Design and Build a Mobile Infant Warmer Using a DC Heater," Int. Res. J. Eng. Technol., vol. 07, no. 02, pp. 145-152, 2019.

[13] E. W. Sinuraya and R. J. Pamungkas, "Design of Temperature Control System for Infant Incubator using Auto Tuning Fuzzy-PI Controller," Biomed. Eng. Int. vol. 3, no. 1, pp. 33-41, 2019.

[14] American College of Obstetricians and Gynecologists, "The Apgar Score: Committe Opinion No 644," Obstet. Gynecol., vol. 126, no.
644, pp. e52-55, 2015, [Online].

[15] A. Nurwanto, E. Y. St, A. Kholiq, and S. S. T. Mt, "Modified Infant Warmer Equipped with Phototherapy (Skin Sensor and Phototherapy Parameters)," IOP Conf. Ser. vol 2, no 2 pp. 1-10, 2006.

[16] P. Jagadeesh, G. Karthick, K. Reddy, and S. V. Reddy, "Design and Development of an Inexpensive Temperature Controller for an Infant Incubator," Int. J. Adv. Res. Electr. Electron. Instrum. Eng. (An ISO, vol. 3297, pp. 10194-10201, 2007, [Online]. Available: www.ijareeie.com.

[17] Y. Molgat-Seon, T. Daboval, S. Chou, and O. Jay, "Accidental overheating of a newborn under an infant radiant warmer: A lesson for future use," J. Perinatol., vol. 33, no. 9, pp. 738-739, 2013, doi: 10.1038/jp.2013.32.

[18] H. Jadav, A. Bansode, and P. D. Sharma, "PID Temperature Controller Infant Incubator Using RTD," Int. Res. J. Eng. Technol., vol. 11, no 3, pp. 13-16, 2018.

[19] K. Roongprasert, P. Phasukkit, S. Airphaiboon, C. Pintavirooj, N. Thongpance, and A. Sanpanich, "Heat transfer efficiency analysis of infant radiant warmer by 3D finite element method," 5th 2012 Biomed. Eng. Int. Conf. BMEiCON 2012, pp. 4-7, 2012, doi 10.1109/BMEiCon.2012.6465474.

[20] W. Widhiada, I. N. G. Antara, I. N. Budiarsa, and I. M. G. Karohika, "The Robust PID Control System of Temperature Stability and Humidity on Infant Incubator Based on Arduino at Mega 2560," IOP Conf. Ser. Earth Environ. Sci., vol. 248, no. 1, 2019, doi: $10.1088 / 1755-1315 / 248 / 1 / 012046$

Attachment:

a. Schematic+Board:

https://drive.google.com/drive/folders/13iylYgpucehQRqm0mMDQ uZl1bATPqTPz?usp=sharing

b. Listing Program :

https://drive.google.com/drive/folders/1RM5iLuNIPfAqiQx5PAltXM75PVIfnx1?usp=sharing 